\title{
Polarity Switch within Social Networks
}

\author{
Sara Abas*, Malika Addou, Zineb Rachik \\ Architecture, System and Networks Team (ASYR) - Laboratory of Systems Engineering (LaGeS), Hassania School of Public Works \\ EHTP, Casablanca, 20230, Morocco
}

\begin{tabular}{l} 
A R T I C L E I N F O \\
\hline Article history: \\
Received: 27 June, 2020 \\
Accepted: 21 October, 2020 \\
Online: 27 November, 2020 \\
\hline
\end{tabular}

Keywords:

Online Users

Positive Polarity

Propagation of Information

Social Networks

\begin{abstract}
A B S T R A C T
It is the age of information. Social networks are the main reason why, following the increasing activity of online users. With this comes a big impact on the real world, it can be positive and highly negative as well. Therefore, research in this field is highly needed for the betterment of societal behaviors within social networks, to minimize the negative impact. When a negatively polarized message spreads over time, it is eventually exposed to most users of that social network where it is spreading. Social network communities start behaving in a negatively polarized way, due to the negative nature of the propagation of information. In order to optimize technological intervention to the benefit of humanity, in this paper an approach is elaborated in order to help switch the dominant polarity in the case of negativity. The platform of reference is Twitter, since it classifies trends per area, which polarities' dominate within the social network. To achieve that, an extraction as well as a sentiment analysis method are developed to initiate the positive polarity introduction in the network. The idea behind introducing a positive polarity in a network, relies on the studies of the positive effect nature has on users. The positively polarized promoted content is selected from sponsored content of users who consent to spreading their posts.
\end{abstract}

\section{Introduction}

In the year 2020, certain events agitated the users of social media platforms, such as: the Covid pandemic and the American social justice riots. As a result, fear spread across the globe not only virtually but also in the real world. This rapid propagation of this type of information has resulted in the spread of misinformation, hatred, cyber-bullying and other unpleasing social behaviors, leading to real life damages. This is where the work of the present paper comes in handy. It becomes unmanageable when the information spreads vastly and one of the key ones who can change this, are the propagators themselves. A study shows that users whose mood shifts are mostly negative generate rants, which are angry forms of expressions [1]. In the case of reaching a big audience, their angry content spreads all over the social platform. As an attempt to reduce the bad effect such users have on those who share the same platform, this paper utilizes research on how nature calms the mood. To promote nature photos or videos on the feed of the potential disruptive users, the selected visual content is extracted form the sponsored data. Sponsored data is data whose owners give consent of publication to online users of the platform. Users who promote their content target people according to many

${ }^{*}$ Corresponding Author: Sara Abas, Email: sara9abas@gmail.com criteria, such as gender, age, geographic area, or center of interests. This paper suggests adding to these criteria, in case the content is nature related, the disruptive users in order to also reach them. To intervene positively; this research suggests a method whose end goal is to switch the user's mood to a positive optimistic one, so that the negative content is less likely to be shared. The article's suggested approach evaluates the current or recently published trends on the Twitter platform, in order to identify the polarity of the dominant news. After this phase, the best contributors in terms of propagation of negatively polarized information are selected. Comes then the procedure of providing them with the polarity switch method as described in the third section of this article. This paper is divided into four sections. The first one is the introduction, the second one is the state of the art in order to mention the previous works. The third one describes the proposed method. The fourth one provides a conclusion.

\section{State of the Art}

Social media has proven itself to be a fast-growing platform for social exchange [2]. When users express themselves on social media, they insinuate approval, disapproval or a neutral statement. To translate their content polarity wise, the process of sentiment analysis helps. In order to achieve that in the proposed method, 
Natural Language Processing tools are referred to for structural analysis.

\subsection{Natural Language Processing}

The NLP is an artificial intelligence processing language to create algorithms that can translate human language. Most commonly used NLP platforms are: NLTK (Natural Language Toolkit) [3], spaCy [4], Stanford's NLP tools [5], including the part-of-speech tagger [6], the named entity recognizer [7], the parser, and other provided components.

\subsection{Sentiment Analysis}

\subsubsection{Existing approaches}

The rich amount of user-provided data is a gift from social media to content analysis studies. Sentiment analysis, is the study of emotions behind textual content. Review sites are a good example of data made available for research purposes. They are reflective of the clients' receptivity of any provided products. This data source is used for the purpose of testing sentiment analysis solutions. In the available literature, some opinion mining studies investigated different approaches. Turney's Algorithm [8] is a clear example of an unsupervised method for sentiment analysis. The authors use identify the polarity of a phrase, using the Alta Vista [9] as a Web Search Engine resource.

A positive polarity is defined by the identification of the phrase with the word "excellent". Similarly, a polarity, which is classified as negative, is calculated by the co-existence of a phrase with the word "poor".

$\mathrm{SO}$ (phrase) = hits(phrase NEAR “excellent") hits("poor") $\log 2$ hits(phrase NEAR “poor") hits(“excellent”)

The hits(phrase NEAR "excellent") "NEAR" operator in the equation represents the co-occurrence of " excellent" and the sentence itself, via the number of search results in Alta Vista. This method is estimated to be $74 \%$ accurate. However, there are many words which have different semantic meanings but are spelled the same in the sequence of letters. In this case, this method is likely to lead to the wrong semantic analysis of words. In this paper, a method which identifies a unique meaning to words in tweets, is presented. However, nowadays on social platforms like Twitter, new words are constantly emerging. The social network's users come up with new terms to exchange, which don't have a meaning prior to their first emergence on the platform. These words can't be found on WordNet, which SentiWordNet relies on in the first place. This paper, presents a solution to these newly invented words as a follow up to the main solution. Such words are not available on external database resources. The commonly used sentiment analysis algorithms are Baseline Algorithm [8], Modified Baseline [9], and Turney's Algorithm [4] which were discussed above. However, they are depend on formal phrasing and they don't take into consideration disambiguation. Other works $[10,11]$ support emoticon analysis, because they are emotionally expressive. An emoticon is a symbol or icon used, to express an emotion without words. The authors' works' relied on a list of pre-classified emoticons or emojis, and emotionally and intensively expressive words. These methods were proven efficient. However, on platforms like Twitter, emoticons are no longer inserted via text, instead they are images provided by the operating system of the phone. A study [12] using available Dutch Tweets, identifies emoticon polarities based on tweets. However, it only studies text-inserted emoticons. Text-inserted emoticons are icons that show up when the user types certain textual character sequences. With the updated phone operating systems of today, users tweet using the available emoticons and do not necessarily type textual symbols.

\subsubsection{Nature's effect}

The more relieved a user is, the less likely they are to spread negativity. Several researches showed that visualizing images of natural views and landscapes, whether they are viewed as 2D photographs, or scenes, resulted in healthy rewinding effects, such as canceling visual and attentional areas of the brain, removing eye blinking and stress [13, 22]. The study [22] investigated 37 articles showing real evidence of the physiological results of witnessing natural views, through on indoor setting experiments-Exposures to display stimuli, confirmed that viewing natural scenery created a physiological relaxing state.

\section{Contribution}

The aim of the present approach is to select the social network's propagators which spread negativity within the network. Negativity comes from a content which is negatively polarized, and doesn't align with the positive spectrum of human emotions like: peace, harmony, laughter, love etc. The reason behind detecting the users who spread negativity, is to expose them to nature images, in order to reduce their stress levels. In this work, the social platform Twitter is used for its available "Trends" functionality, which is going to help isolate the most controversial topics, and therefore the most controversial users who discuss these topics. In order to achieve this and to carefully extract trends accordingly to their audience and propagators, the country is first selected on the social platform. The trends functionality is defined by the selection of the geographic area on the Twitter platform. So instead of studying random trends, Twitter selects trends per country. The trends functionality is defined by the selection of the geographic area on the Twitter platform. This helps filter information and circle the social network in which trends are spreading. Once this is achieved, the geographic area where an information is spreading most is considered the social network of base, on which the suggested methods of this paper will run.

\subsection{Suggested approach}

The macroscopic method is summed up in the figure below.

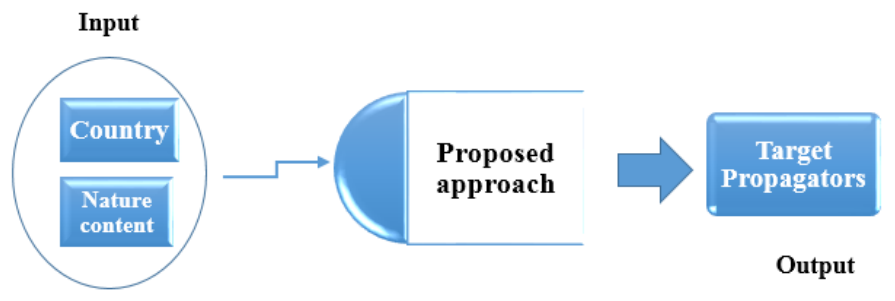

Figure 1: Explained Method Macroscopically

In the entry of the final system, first the country and the nature related sponsored data is provided. This automatically zooms the focus on the content shared within the social network related to that country. As for the nature content, the sponsored database 
content is selectively pre-extracted from the sponsored database content of the paltform. On the social network platform, some pages posts are sponsored in order for the page to be marketed and reach a broad audience. From the sponsored content, text mining to find the following keywords is applied: $\mathrm{K}=\{$ Nature ; Landscape ; Ocean ; Mountain ; Flower ; River ; Beach ; Tree ; Forest ; Sea ; Waterfall $\}$. If the sponsored image's caption contains a word that belongs to the set $\mathrm{K}$, it is a potential advertised content to the target propagators. From this set, the top 20 liked images are chosen, in order to optimize the chance of having a positive influence on the selected users' moods.

After running the method, the final result is a set of target propagators, to whom the selected nature data is displayed, which is sponsored by the marketers of social media seeking promotion of their nature related pages.

\subsubsection{Detailed Method}

After the country is selected, the process of the suggested method follows four steps.

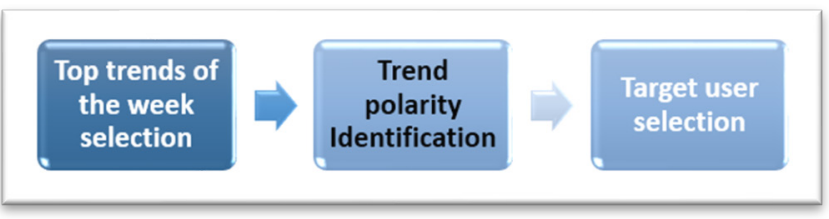

Figure 2: Detailed Approach

Phase 1: The first phase as illustrated in fig2 is the top trends of the week selection. In this first phase, a simple query on the platform retrieves the top 20 trends of the day, listed in the country: https://twitter.com/i/trends. On the social platform, the top 20 trends are selected and highlighted per country. However, a trend yesterday could be completely neglected today, and no longer have consistent impact. In order to identify the consistently impactful trends, a method is developed that calculates trend relevancy over the past 7 days. The variable "Real_Rank" reflects the real rank of the trend, according to the past 7 days on Twitter.

Let a be one of the top 20 trends of today.

$$
\operatorname{Real} \_\operatorname{Rank}(a)=\sum_{0}^{6} \frac{7-j}{\operatorname{Rank}(a ; t o d a y-j)}
$$

Phase 2: Trend polarity Identification. It is necessary to identify which one of the top trends from the first phrase, negatively impacts the social network. In order that, a sentiment analysis method is developed to determine the polarity behind the trend. A trend $\mathrm{Tr}$ is a dynamic set of content, which changes every second. In order to identify the polarity of a trend the global polarity of its components is evaluated.

$\operatorname{Tr}(j)=\left\{T W_{1} ; T W_{2} ; . . T W_{n}\right\} \mathrm{j}$ is the current day on which the algorithm runs. To identify the polarity of a single tweet Tw1, which is the shared post, the POS Tagger is used in order to retrieve the mentioned adjectives in the sentence. Databases for research purposes are fortunately made available byplatforms, such as Amazon. This includes reviews (ratings, text, helpfulness votes).

Let the following be tweets about the Trend "\#destroyThings" extracted from the first phase the platform.

"It is time to destroy things because we hate this horrible system." $\left(\mathrm{Tw}_{1}\right)$
"Destroying is dangerous. Please refrain from doing this bad habit" $\left(\mathrm{Tw}_{2}\right)$

In this sample example there are 4 reviews.

Review $1:$ I hate this audiobook.

Review 2 : I don't like it

Review 3 : Boring.

Review 4 : Not very nice.

(Review 1, 0 stars), (Review 2, 2 star), (Review 3, 1 stars),

(Review 4, 0.5 stars).

Clearly, the stars given by the reviewers who labelled their review with "horrible" aren't passed 3 stars.

We use the identification method of the polarity of phrase method.

The positivity rate is defined as follows:

$$
\text { Pos }(\text { Trend })=\frac{\sum \text { Polarity }(\text { phrase } k)}{N}
$$

$\mathrm{N}$ is the number of tweets on the trend "Trend" list.

For adjectives that newly emerge on the social platform, the same method is used on the acknowledged adjectives by the POS Tagger, which occur on their same sentence. In order to explain this, the following examples are cited.

"Amazing. This movie is epic"

"Glad I watched this. Epic."

"This cat is epic. So funny. The owner is odd."

From the first tweet, "amazing" is the identified adjective. This word has a positive polarity. The same applies for the second statement. For the third one, the word "odd" is singled out.

In order to identify the polarity of a newly emerging word, which is a word that does not exist on available knowledge base, the following equation is used. If $\mathrm{wk}$ is a positively polarized word, then $b k=1 . \mathrm{bk}(\mathrm{w} 1)$ represents an adjective that co-occurs with the word $\mathrm{w}_{1}$ in the same phrase, $\mathrm{m}$ is the number of adjectives in the same tweet $\mathrm{j}$.

$$
\begin{gathered}
P_{-} t w e e t_{-} j\left(W_{1}\right)=\frac{\sum b k(w 1)}{m} \\
\operatorname{Polarity}(w k)=\frac{\sum P_{-} t w e e t_{-} j(w k)}{N}
\end{gathered}
$$

where $\mathrm{N}$ is the number of tweets containing the word w1.

Phase 3: In this phase, finally the target disruptive users of the social network are selected.

If $\operatorname{Pos}$ (Trend) $<50 \%$ after it has been selected as a top ranking trend from the first phase, the top 10 most liked tweets belonging to the set of this trend are selected, and the propagators of these tweets are targeted by the selected nature sponsored images.

\subsubsection{Algorithm}

The global algorithm for the selection of the users to whom nature's sponsored data is exposed is described as follows below. Let $\mathrm{T}$ be the social network, and $\mathrm{C}$ the country selected in the entry of the system.

\begin{tabular}{l}
\hline Algorithm 1: procedure target_Selection \\
\hline Result: $\mathrm{P}$ \\
$\mathrm{S}<-\varnothing, \mathrm{A}<-\varnothing, \mathrm{R}<-\varnothing, \mathrm{P}<-\varnothing$
\end{tabular}


$\mathrm{S}=$ calculate $\operatorname{trendRank}(\mathrm{T})$

$\mathrm{A}=$ polarity_analyze $(\mathrm{S})$

$\mathrm{R}=\operatorname{select}(\mathrm{A}, 10)$

$\mathrm{P}=$ select_propagator $(\mathrm{R})$

Calculate trendRank(T) is a result set which contains 20 vectors. $\mathrm{C}$ is the country. In each vector are stored: the trend reference and the real rank of the trend T calculated throughout 7 days, which is calculated by the equation (1). Only trends that are related to the top 20 values of the equation are stored.

Polarity_analyze(S), is a result set which contains a set of vectors. In each vector are stored the trend reference, and the polarity of the trend. The trends are the ones contained in S. The polarity of each trend is calculated via the equation (8).

Select $(A, 10)$ is a method that extracts the top 10 negatively polarized trends from the set $\mathrm{A}$, and results in a set $\mathrm{R}$.

Select_propagators() method uses the set $\mathrm{R}$ in order to extract the top 10 most liked posts in the set, and simply identify their users.

\section{Conclusion}

In the aftermath of a controversial event, social media is usually the most accountable for the chaotic spread of information as well as misinformation. This paper suggests a new method to relieve the online stress, and therefore the real world second effects. In order to do that, available research is used on how nature changes one's emotional state, and calms it. The approach aims to extract the target propagators that need to be stress relieved the most in order to reduce the chaotic information propagation. The goal is to promote nature displays on their timelines. These displays are selected from users whose content is sponsored for promotion. On top of their usual targets, the platform's disruptive users are added in their target potential clients. In order to do that, a sentiment analysis method is developed and information from the social platforms is extracted. In the future works, other aspects are to be covered such as the use of emoticons, the observation of longterm effects of the algorithm on the main propagators and their respective social networks.

\section{Conflict of Interest}

The authors declare no conflict of interest.

\section{References}

[1] R. Martin, K. Coyier, L. Vansistine, K. Schroeder, "Anger on the Internet: The Perceived Value of Rant-Sites, " Cyberpsychology, behavior and social networking, 16(2), 119-122, 2013, doi: 10.1089/cyber.2012.0130.

[2] E. Muller, R. Peters, "The effect of social networks structure on innovation performance: A review and directions for research, " International Journal of Research in Marketing, 36(1), 3-19, 2019, doi: 10.1016/j.jiresmar.2018.05.003.

[3] A. Hamandi, A. AbdulHassan, H. Bahjat, "Natural Language Processing Using Natural Language ToolKit," Iraqi Journal of Information Technology, 7(2), 2016.

[4] https://spacy.io/

[5] https://nlp.stanford.edu/software/

[6] A. Voutilainen, Part-of-Speech Tagging, The Oxford Handbook of Computational Linguistics, 2012.

[7] https://nlp.stanford.edu/software/CRF-NER.html
[8] P. Turney, "Thumbs Up or Thumbs Down? Semantic Orientation Applied to Unsupervised Classification of Reviews," in Proceedings of the 40th annual meeting on association for computational linguistics, 417-424, 2002.

[9] B. Pang, L. Lee, S. Vaithyanathan, "Thumbs up? Sentiment Classification using Machine Learning Techniques," in Proceedings of the 2002 Conference on Empirical Methods in Natural Language Processing, 79-86, 2002.

[10] S.Das, M. Chen, "Yahoo! for amazon: Extracting market sentiment from stock message boards," in 8th Asia Pacific Finance Association Annual Conference, 2001.

[11] D. Cirqueira, M. Fontes Pinheiro, A. Jacob, F. Lobato and A. Santana, "A Literature Review in Preprocessing for Sentiment Analysis for Brazilian Portuguese Social Media," in 2018 IEEE/WIC/ACM International Conference on Web Intelligence (WI), Santiago, Chile, 746-749, 2018, doi: 10.1109/WI.2018.00008.

[12] A. Hogenboom, D. Bal and F. Frasincar, "Exploiting Emoticons in sent iment analysis," in Proceedings of the 28th Annual ACM symposium on Applied Computing, 703-710, 2013.

[13] K. Gwang-Won, J. Gwang-Won, K. Tae-Hoon, . “Functional neuroanatomy associated with natural and urban scenic views in the human brain:3.0 T functional MR imaging," Korean Journal of Radiology, 11(5), 507-513, 2013, doi: 10.3348/kjr.2010.11.5.507.

[14] K. Gwang-Won, J. Gwang-Won, K. Tae-Hoon, . "Human brain activation in response to visual stimulation with rural and urban scenery pictures: a functional magnetic resonance imaging study," Sci Total Environ, 408(12), 2600-7, 2010, doi: 10.1016/j.scitotenv.2010.02.025.

[15] K. Laumann, T. Gärling, K. Stormark, "Selective attention and heart rate responses to natural and urban environments," Environmental Psychology, 23, 125-134, 2003.

[16] C.Y. Chang, W.E. Hammitt, P.K. Chen, Machnik, "Psychophysiological responses and restorative values of natural environments," in Taiwan. Landscape. Urban Plan, 85, 79-84, 2008.

[17] V.F. Gladwell, DK. Brown, JL. Barton, "The effects of views of nature on autonomic control, "European Journal of Applied Physiology, 112(9), 33793386, 2012, doi: 10.1007/s00421-012-2318-8.

[18] D.K. Brown et al., "Viewing nature scenes positively affects recovery of autonomic function following acute-mental stress," Environ. Sci. Technol. 47, 5562-5569, 2013.

[19] M. Igarashi, T. Yamamoto, J. Lee, C. Song, H. Ikei, Y. Miyazaki, "Effects of stimulation by three-dimensional natural images on prefrontal cortex and autonomic nerve activity: A comparison with stimulation using twodimensional images," Cognitive Processing, 15, 551-556, 2014.

[20] A.P. Anderson, M.D. Mayer, A.M. Fellow, D.R. Cowan, M.T. Hegel, J.C. Buckey, "Relaxation with immersive natural scenes presented using virtual reality," Aerospace Medicine and Human Performance, 88, 520-526, 2017.

[21] H. Jo, C. Song, Y. Miyazaki, "Physiological Benefits of Viewing Nature: A Systematic Review of Indoor Experiments," Int J Environ Res Public Health, 16(23), 4739, 2019, doi: 10.3390/ijerph16234739.

[22] C. Diamantini, A. Mircolli, D. Potena, " A Negation Handling Technique for Sentiment Analysis," in 2016 International Conference on Collaboration Technologies and Systems (CTS), 188-195, 2016, doi: 10.1109/CTS.2016.0048. 